\title{
A GENERALIZED MATHARU-AUJLA INEQUALITY
}

\author{
SIYUAN SHEN, JUNMIN HAN AND JiAN SHI
}

Abstract. In this paper, we will show a generalized Matharu-Aujla log majorization inequality via an operator order preserving inequality, which extends the related results.

Mathematics subject classification (2010): 47A63.

Keywords and phrases: Log majorization, generalized Furuta inequality, Matharu-Aujla inequality.

\section{REFERENCES}

[1] T. ANDo, F. HiAI, Log majorization and complementary Golden-Thompson type inequality, Linear Algebra Appl. 197 (1994), 113-131.

[2] T. FURUTA, An extension of order preserving operator inequality, Math. Inequal. Appl. 13, 1 (2010), 49-56.

[3] T. FURUTA, Extensions of inequalities for unitarily invariant norms via log majorization, Linear Algebra Appl. 436 (2012), 3463-3468.

[4] F. Kubo, T. Ando, Means of positive linear operators, Math. Ann. 246 (1980), 205-224.

[5] J. S. Matharu, J. S. Aujla, Some inequalities for unitarily invariant norms, Linear Algebra Appl. 436 (2012), 1623-1631. 